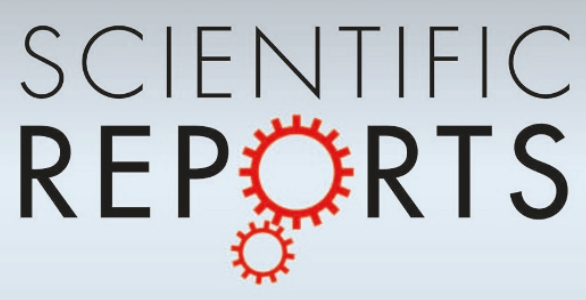

OPEN

\section{SUBJECT AREAS:}

ELECTRICAL AND ELECTRONIC ENGINEERING

APPLIED PHYSICS

ELECTRONIC AND SPINTRONIC DEVICES

Received

30 May 2014

Accepted

2 December 2014

Published

15 January 2015

Correspondence and requests for materials should be addressed to

J.Z.W. (jizheng@ iccas.ac.cn)

\title{
Uncovering the role of cathode buffer layer in organic solar cells
}

\author{
Boyuan Qi ${ }^{1}, 2$, Zhi-Guo Zhang' \& Jizheng Wang'
}

'Beijing National Laboratory for Molecular Sciences, Key Laboratory of Organic Solids, Institute of Chemistry, Chinese Academy of Sciences, Beijing 100190, P.R. China, ${ }^{2}$ Graduate University of Chinese Academy of Sciences, Beijing 100049, P. R. China.

Organic solar cells (OSCs) as the third generation photovoltaic devices have drawn intense research, for their ability to be easily deposited by low-cost solution coating technologies. However the cathode in conventional OSCs, Ca, can be only deposited by thermal evaporation and is highly unstable in ambient. Therefore various solution processible cathode buffer layers (CBLs) are synthesized as substitute of Ca and show excellent effect in optimizing performance of OSCs. Yet, there is still no universal consensus on the mechanism that how CBL works, which is evidently a critical scientific issue that should be addressed. In this article detailed studies are targeted on the interfacial physics at the interface between active layer and cathode (with and without treatment of a polar CBL) by using ultraviolet photoelectron spectroscopy, capacitance-voltage measurement, and impedance spectroscopy. The experimental data demonstrate that CBL mainly takes effect in three ways: suppressing surface states at the surface of active layer, protecting the active layer from being damaged by thermally evaporated cathode, and changing the energy level alignment by forming dipole moments with active layer and/or cathode. Our findings here provide a comprehensive picture of interfacial physics in devices with and without CBL.

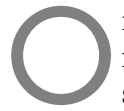
rganic solar cells (OSCs) have attracted a continuously growing interest in the roadmap of clean energy, for their potential to realize ultralow-cost manufacturing and ability to be deposited on various flexible substrates by solution process, such as roll-to-roll coating technology. However there is still a big challenge to overcome for the commercialization of OSCs, i.e., achieving solution processible cathode. In conventional devices the commonly used cathode is thermally evaporated $\mathrm{Ca} / \mathrm{Al}$. But $\mathrm{Ca}$ is readily oxidized when exposed to the air and moisture, and hence induces large performance drop as time goes on. Therefore cathode buffer layer (CBL), as an inert and solution processible substitute of $\mathrm{Ca}$, is a hot spot in the field of OSCs ${ }^{1-3}$, and a significant achievement in efficiency $(9.2 \%)$ has been made promoted by this research interest ${ }^{4}$. Despite this progress, the precise mechanism by which CBL takes effect in OSCs is still under debate. Currently the most popular strategy of designing CBL molecules is to introduce polar groups into the backbones ${ }^{5-9}$. These polar groups are intended to alter the energy level alignment between the cathode and active layer, increase the built-in potential, and enhance the open-circuit voltage $\left(\mathrm{V}_{\mathrm{OC}}\right)$ and power conversion efficiency (PCE) ultimately. However, a different perspective has come out from the recent observations that even treating active layer with methanol (the solvent to dissolve $\mathrm{CBL}$ ), the $\mathrm{V}_{\mathrm{OC}}$ and PCE of device could be improved drastically compared to the control device $e^{10-14}$, indicating the polarity of CBL may be unnecessary.

In this manuscript, we intend to investigate this important issue. By using a fullerene based polar molecule Nmethylpiperazine adduct $\mathrm{C}_{60}$ (NMPAC) as CBL in high-performance system thieno [3, 4-b]-thiophene/benzodithiophene: [6, 6]-phenyl C71-butyric acid methyl ester (PTB7:PC $\left.{ }_{71} \mathrm{BM}\right), \mathrm{V}_{\mathrm{OC}}$ and PCE are found to be enhanced obviously, which is consistent with previous reports ${ }^{10-14}$. In order to explore the role of molecular polarity, the energy levels of blend surface are measured with UPS. It is observed however that there is no significant change before and after CBL deposition. This implies that NMPAC does not work by means of proving dipole moments to the organic blend. In order to figure out the role of NMPAC, capacitance-voltage (CV) measurements are applied to directly target on the interface between active layer and cathode, and find that the $\mathrm{CV}$ plots show a large discrepancy in dark and under illumination in device without CBL. This discrepancy is attributed to the surface states at the interface between active layer and cathode which lead to a slow kinetics for charge transfer, and as a result non-equilibrium carriers generated under illumination are blocked at the interface. After methanol and CBL treatment, surface states are reduced significantly and discrepancy becomes less with the increasing thickness of CBL. Then the density and energy level of interfacial surface states and lifetime of charge carriers are calculated via CV and impedance measurements. Carriers in devices with CBL treatment suffer less recombination and show longer lifetimes resulting from less surface states. Finally molecular polarity is briefly 
discussed with UPS data and previous reports. It is concluded that dipole moments formed at the interface of blend/CBL and CBL/ cathode both contribute to the overall enhance of $\mathrm{V}_{\mathrm{OC}}$ on condition that surface states are supressed.

\section{Results}

J-V characterization. The NMPAC was obtained via facile synthesis procedures with high yields using eco-friendly reagents according to the method initially described by Nakamura ${ }^{15,16}$, while their application remains largely unknown. Here, our exploration of NMPAC as the CBL is inspired by its unique virtues of being structurally similar to the conventionally used fullerene acceptors (such as PCBM) and most importantly possible dipole moments at the interfaces provided by the four adducted $N$-methyl piperazine polarity groups. Moreover it has been reported that fullerene based materials have similar energy levels with PCBM, which makes them to be energetically favorable for electron extraction ${ }^{17-19}$. The configuration of the OSCs is indium tin oxide (ITO)/poly (3, 4ethylenedioxythiophene): poly(styrenesulfonate) (PEDOT:PSS)/ PTB7:PC ${ }_{71} \mathrm{BM} / \mathrm{CBL}$ (w/wo)/Al (shown in Fig. 1). It should be noted that NMPAC is dissolved in methanol and spin-coated on active layer, which means the active layers in our experiments also have been treated by methanol.

To optimize the thickness of CBL, concentrations of the solution are chosen as $0.5,1.0,1.5$ and $2.0 \mathrm{mg} / \mathrm{ml}$ with the spin-coating speed fixed at 3,000 rpm, and thicknesses are 9, 7, 5 and $3 \mathrm{~nm}$, respectively. The current density-voltage $(\mathrm{J}-\mathrm{V})$ characteristics of devices with and without CBL are shown in Fig. 2. All of the parameters measured are listed in Table 1, it can be seen that short-circuit curent density $\left(\mathrm{J}_{\mathrm{SC}}\right)$ is improved by about $1 \mathrm{~mA} / \mathrm{cm}^{2}$ from the control device to the optimized device, and even with methanol treatment the $\mathrm{J}_{\mathrm{SC}}$ is increased. This is induced by the enhanced optical absorption in devices, and can be proved by the increased external quantum efficiency (EQE) spectra and decreased reflectance spectra shown in Supplementary Fig. S1.

Meanwhile, even treated by methanol, $\mathrm{V}_{\mathrm{OC}}$ varies a lot compared to that of the control device (from $0.519 \mathrm{~V}$ to $0.688 \mathrm{~V}$ ). This can be explained by the reduced dark current. From the J-V curves in dark, two parameters characterizing the property of diodes, namely ideality factor $(\mathrm{n})$ and saturation dark current dentisy $\left(\mathrm{J}_{0}\right)$, can be extracted by fitting the data into Shockley equation listed below (fitting scheme is shown in Fig. 2b and details can be found in the supporting information of ref. 20):

$$
J=\frac{R_{s h}}{R_{s h}+R_{s}}\left\{J_{0}\left[\exp \left(\frac{q\left(V-J R_{s}\right)}{n k_{B} T}\right)-1\right]+\frac{V}{R_{s h}}\right\}-J_{p h}
$$

Here, $\mathrm{q}$ is the elementary charge, $k_{B} T$ the thermal energy, $J_{p h}$ the photocurrent. After simplificaiton, the $\mathrm{V}_{\mathrm{OC}}$ could be deduced as refs 21, 22:
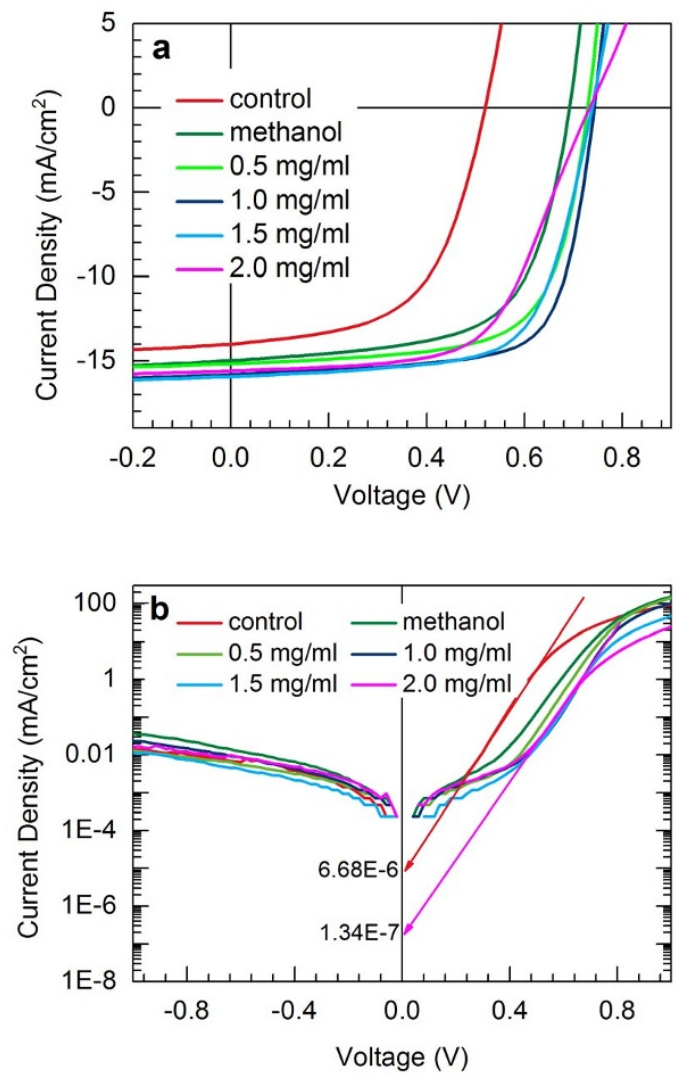

Figure $2 \mid$ Device performance under illumination and in dark. J-V characteristics of devices with methanol treatment and different thickness of CBL: (a) under illumination of AM $1.5 \mathrm{G}, 100 \mathrm{~mW} / \mathrm{cm}^{2}$; (b) in dark. The dashed lines are used to extract the ideality factor and saturation dark current density, by fitting the J-V curves to Shockley equation (only two of them are chosen as examples).

$$
V_{O C}=\frac{n k_{B} T}{q} \ln \left(\frac{J_{S C}}{J_{0}}\right)
$$

Table 1 shows that $\mathrm{J}_{0}$ decreases about 3 orders of magnitude from control device to device with $1.0 \mathrm{mg} / \mathrm{ml}$ CBL. Therefore the $\mathrm{V}_{\mathrm{OC}}$ could be evidently enhanced according to equation (2).

Shunt resistance $\left(\mathrm{R}_{\mathrm{sh}}\right)$ denotes current leakage both from the edge of the cells and inside the device. It has been reported by several groups that hot $\mathrm{Al}$ atoms during thermal evaporation can cause damage to the surface of active layer and diffuse inside the blend ${ }^{23-25}$. Metal atoms are active centers for carriers' recombination and as a result the current loss will increase as reflected by decrease of $\mathrm{R}_{\mathrm{sh}}$. In
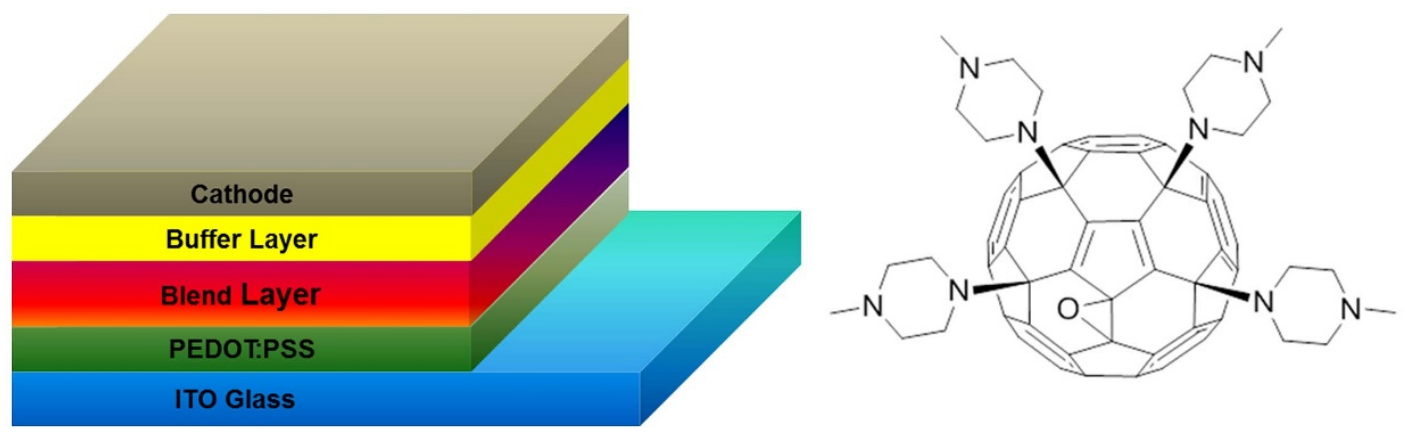

Figure $1 \mid$ Device configuration and chemical structure of NMPAC. Device structure using NMPAC as the cathode buffer layer. 
Table 1 | Device performance and extracted parameters with different CBL under 1 sun illumination

\begin{tabular}{|c|c|c|c|c|c|c|c|c|c|}
\hline Device & $\mathrm{V}_{\mathrm{OC}}[\mathrm{V}]$ & $\mathrm{J}_{\mathrm{SC}}\left[\mathrm{mA} / \mathrm{cm}^{2}\right]$ & $\mathrm{FF}[\%]$ & PCE [\%] & Avg. PCE [\%] ${ }^{a)}$ & $\mathrm{R}_{\mathrm{s}}\left[\Omega \mathrm{cm}^{2}\right]$ & $\mathrm{R}_{\mathrm{sh}}\left[\mathrm{k} \Omega \mathrm{cm}^{2}\right]$ & n [n.a.] & $\mathrm{J}_{0}\left[\mathrm{~mA} / \mathrm{cm}^{2}\right]$ \\
\hline Control & 0.519 & 14.62 & 57.0 & 4.34 & 4.22 & 7.80 & 412.5 & 1.51 & $6.68 \times 10^{-6}$ \\
\hline Methanol & 0.688 & 14.96 & 64.2 & 6.62 & 6.55 & 5.25 & 557.4 & 1.68 & $1.47 \times 10^{-6}$ \\
\hline $0.5 \mathrm{mg} / \mathrm{ml}$ & 0.726 & 15.15 & 68.3 & 7.51 & 7.37 & 4.36 & 897.5 & 1.54 & $1.61 \times 10^{-7}$ \\
\hline $1.5 \mathrm{mg} / \mathrm{ml}$ & 0.737 & 15.92 & 67.5 & 7.92 & 7.72 & 7.08 & 831.3 & 1.48 & $2.80 \times 10^{-8}$ \\
\hline $2.0 \mathrm{mg} / \mathrm{ml}$ & 0.733 & 15.59 & 60.1 & 6.87 & 6.74 & 15.2 & 992.5 & 1.62 & $1.34 \times 10^{-7}$ \\
\hline
\end{tabular}

$V_{O C}$, open-circuit voltage; $J_{S C}$, short-ciucuit current density; FF, fill factor; PCE, power conversion efficiency; $R_{s}$, series resistance; $R_{\text {sh, }}$ shunt resistance; $n$, ideality factor; $J_{s}$, reverse saturation dark current density.

a)Avg. PCE is average PCE for 8 devices.

this work, CBL has been shown to be beneficial to prevent cells from current loss. After spin-coating CBL, $\mathrm{R}_{\mathrm{sh}}$ increases dramatically and remains independent to the thickness of CBL. Additionally a certain thickness of NMPAC layer can reduce $\mathrm{R}_{\mathrm{s}}$ effectively. This could be explained from the point of view of energy levels. From cyclic voltammogram shown in Supplementary Fig. S2, NMPAC has the highest occupied molecular orbital (HOMO) level of $-5.73 \mathrm{eV}$ (lower than that of PTB7, $-5.2 \mathrm{eV}$ ) and lowest unoccupied molecular orbitals (LUMO) level of $-3.86 \mathrm{eV}$ (similar to that of $\mathrm{PC}_{71} \mathrm{BM}$, $-3.9 \mathrm{eV})$. Therefore a certain thickness of NMPAC layer can block holes without obstructing extraction of electrons, so $R_{s}$ can be effectively reduced. However, if NMPAC layer is too thick, electrons should travel a long way to reach the cathode compared to holes, this may induce charge accumulation inside the device and hinder the transport of carriers. Therefore when concentrations of CBL change from optimized 1.0 to $2.0 \mathrm{mg} / \mathrm{ml}, \mathrm{R}_{\mathrm{s}}$ will increase rapidly from 3.95 to $15.2 \Omega \mathrm{cm}^{2}$. In OSCs both $\mathrm{R}_{\mathrm{s}}$ and $\mathrm{R}_{\mathrm{sh}}$ are important factors influencing $\mathrm{FF}^{26}$, since $\mathrm{R}_{\mathrm{sh}}$ is stable for the devices with CBL, variation of $R_{s}$ is the reason that $F F$ increases from $57 \%$ (for the control device) to the maximum $71.3 \%$ (for the device with $1.0 \mathrm{mg} / \mathrm{ml} \mathrm{CBL}$ ) and then decreases to $60.1 \%$ (for the device with $2.0 \mathrm{mg} / \mathrm{ml} \mathrm{CBL}$ ). In Supplementary Fig. S3 and Table S1, J-V characterization of conventional device (with $\mathrm{Ca} / \mathrm{Al}$ cathode) is also listed for comparison.

Surface states. In order to explore the effect of polarity of NMPAC, three samples are fabricated and measured by UPS, with the structure of: ITO/PEDOT:PSS, ITO/PEDOT:PSS/PTB7:PC 71 BM and ITO/ PEDOT:PSS/PTB7:PC ${ }_{71}$ BM/NMPAC, respectively. Fig. 3a shows the secondary electron cutoff region of the three samples, from which the work function (WF) can be determined. The WF of PEDOT:PSS is deduced to be $5.1 \mathrm{eV}$, in good agreement with its common value. Interestingly the WF of blend shows little change after CBL spin-coated on its surface, opposite to our initial expectations. This suggests that overall dipole moments provided by the CBL are zero at the interface of blend/CBL, maybe the CBL molecules distribute dispersedly in the film, and therefore the dipole moments are counteracted ultimately.

Then CV measurements are performed on the actual devices in dark, with the results of which the built-in potential can be extracted by fitting CV plots into Mott-Schottky equation. From Fig. 4, it is demonstrated that the built-in potential shows an obvious difference between the devices with and without methanol treatment. Here it is assumed that there are surface sates existed at the interface, leading to lower built-in potential in control device. Generally at the surface of crystal, periodic potential field being broken off, adsorption of other kinds of molecules or existence of oxide film can all induce additional energy levels in the band gap, which are called surface state ${ }^{27}$. Similarly disorder at the surface of organic semiconductor can also lead to localized sates into the band gap. What's more, when hot metal atoms are deposited onto the surface of blends, it may also induce extra surface states.
To verify this hypothesis, we apply the CV measurements on devices again under illumination and find the built-in potential decreases under illumination without CBL or methanol treatment, reflected by the left-shift Mott-Schottky plots. Because the Fermi level of surface states lies deep in the bandgap of blends, electrons at the conduction band prefer to fill the surface states at the contact and then the exchange of charge carriers between electrode and semiconductor is mainly accomplished via surface states. Because this charge transfer process is slow, carriers will accumulate at the contact and induce undesirable surface recombination and poor extraction property. Especially under illumination photo-generated excitons provides a large amout of non-equilibrium carriers, highly efficient surface recombination is expected to occur at the interfacial layer. Surface states will get more charges with respect to the dark conditions, due to the slow charge transfer kinetics, too. It leads to an
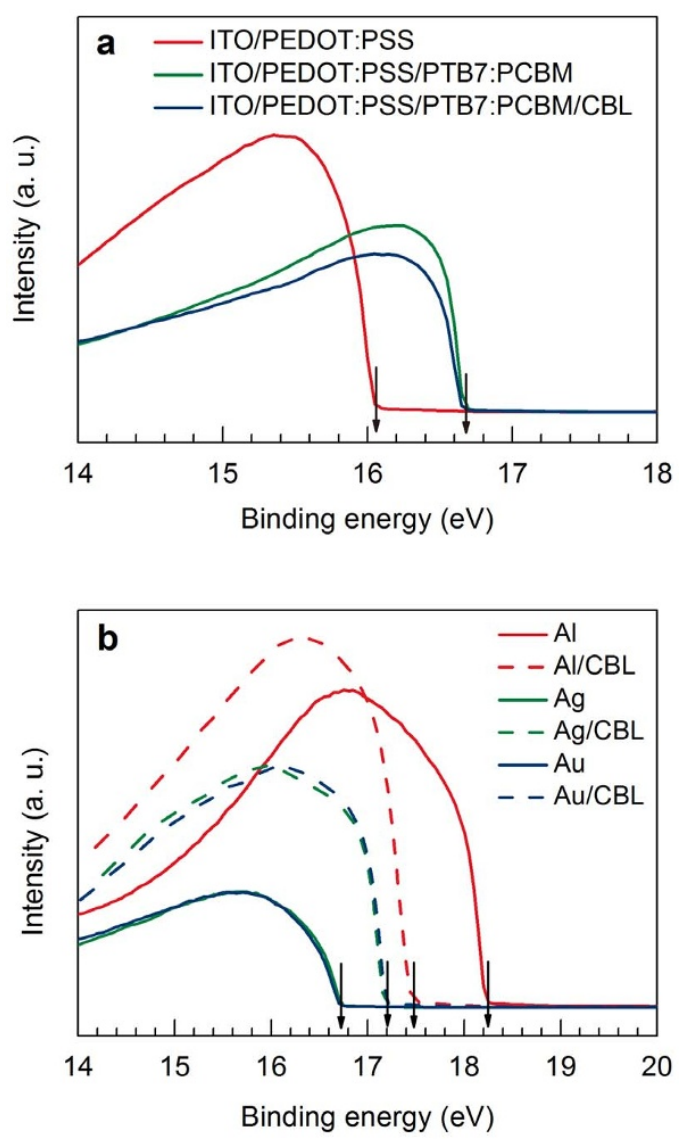

Figure 3 UPS spectra. UPS spectra of CBL on the surface of (a) active layer and (b) various metals. The spectra show the energy region of the secondary electron cut-off and are normalized to the peaks of ITO/ PEDOT:PSS in (a) and Ag/CBL in (b). 

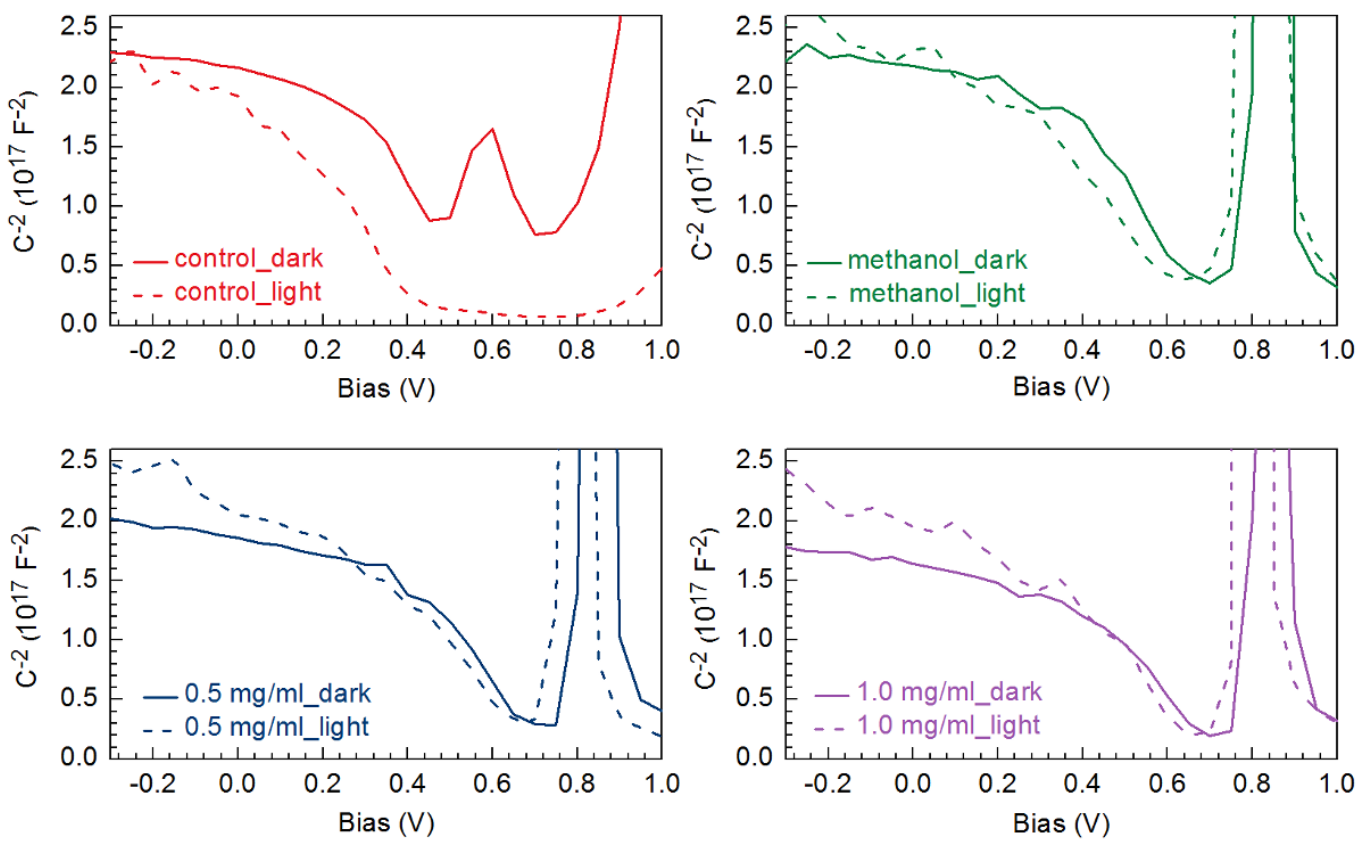

Figure $4 \mid$ Mott-Schottky fitting with CV data. Mott-Schottky plots of devices with different CBL in dark and under 1 sun illumination. From which the $\mathrm{V}_{\mathrm{b}}$ and $\mathrm{N}_{\mathrm{A}}$ can be extracted.

upward of band edges and at the same time the potential drop across the interfacial layer increases. This means the flat-band voltage decreases and could be observed in the Mott-Schottky plots that the position of $\mathrm{V}_{\mathrm{b}}$ moves to the left ${ }^{28,29}$. While if surface states are shallow ones in the bandgap, the kinetics of charge transfer will be fast and the displacement of Mott-Schottky plots will be little or none. From Fig. 4 it is observed that without CBL or methanol treatment the $\mathrm{V}_{\mathrm{b}}$ varies about $0.14 \mathrm{~V}$, proving that there are surface states at the cathode contact; after the methanol treatment the variation of $\mathrm{V}_{\mathrm{b}}$ decreases to only $0.04 \mathrm{~V}$, indicating that surface states could be eliminated effectively by methanol wash; if $1.0 \mathrm{mg} / \mathrm{ml}$ NMPAC is used as the CBL, there is no shift of $V_{b}$ which implies this CBL can protect active layer from being damaged by metal evaporation.

Here we further investigate the surface states by CV and impedance measurements, and discuss it with a well-known device model proposed by Bisquert et al..$^{30}$. Generally the conjugated polymer is $\mathrm{p}$ doped when exposed to air or moisture. After donor and acceptor being blended, the whole active layer is assumed as p-type semiconductor rather than insulator. So the Fermi level of blend is in the vicinity of HOMO level of donor. When low WF metal is deposited onto the surface of active layer, band bending occurs and Schottky barrier is formed (shown in Fig. 5a). Here the contact of anode is seemed as ohmic contact for the similar Fermi levels of blend and PEDOT:PSS layer. From the device model, the height of the built-in barrier is determined by the Fermi level difference between cathode $\left(\phi_{c}\right)$ and blend $\left(E_{F 0}\right)$ :

$$
q V_{b}=E_{F 0}-\phi_{c}
$$

The barrier height could be obtained by applying standard MottSchottky analysis to the results of CV measurements. In order to explore the relationship between WF and barrier height of organic blend, we made solar cells with three different metals, $\mathrm{Ca}, \mathrm{Al}$ and $\mathrm{Ag}$. The structure of the devices is ITO/PEDOT:PSS/blend/metal and their Mott-Schottky plots are shown in Fig. 6. It can be seen $V_{b}$ changes little with different metals. Especially with $\mathrm{Ca}$ and $\mathrm{Al}$ as cathode, despite WF varies from $2.7 \mathrm{eV}$ to $4.2 \mathrm{eV}, \mathrm{V}_{\mathrm{b}}$ changes only
$0.09 \mathrm{~V}$. This is quite opposite to the theory of Schottky that the barrier height is equal to the WF difference between metal and semiconductor. However it has been found that in inorganic devices the barrier height of metal-semiconductor systems is related to the surface states of semiconductor. If the variation of $\mathrm{V}_{\mathrm{b}}$ with $\mathrm{WF}$ of metals is defined with a slope, $S$, then according to the discussion above, if $S=1$, there is no surface states; while if $S=0$, the density of surface states is large and the Fermi level of metal will be totally pinned to
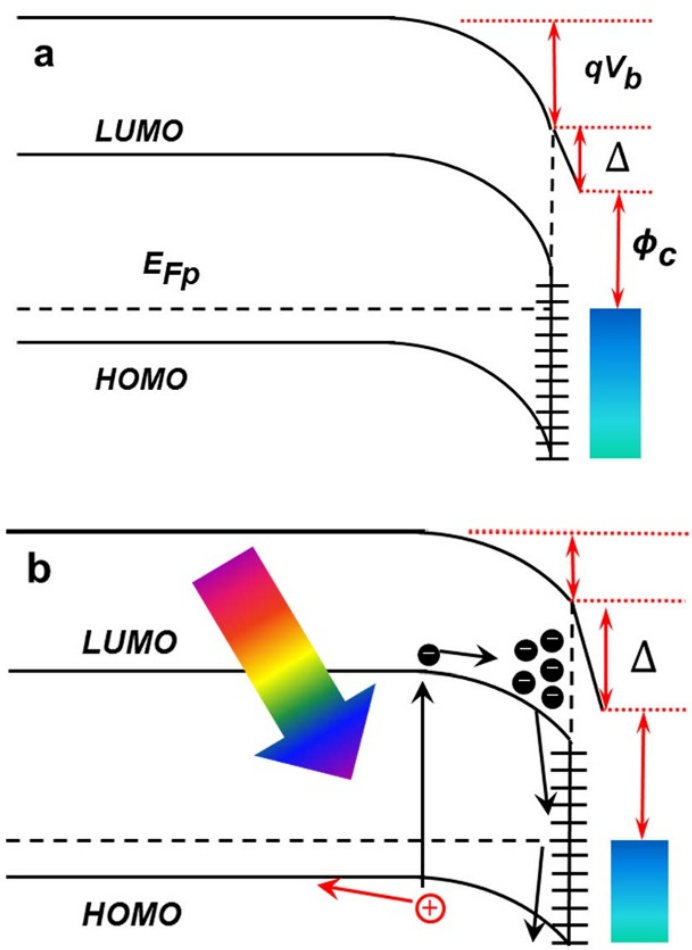

Figure 5 Schematic diagram of energy level and surface states. Energy level alignment of active layer, surface states and metal (a) in dark and (b) under illumination. 


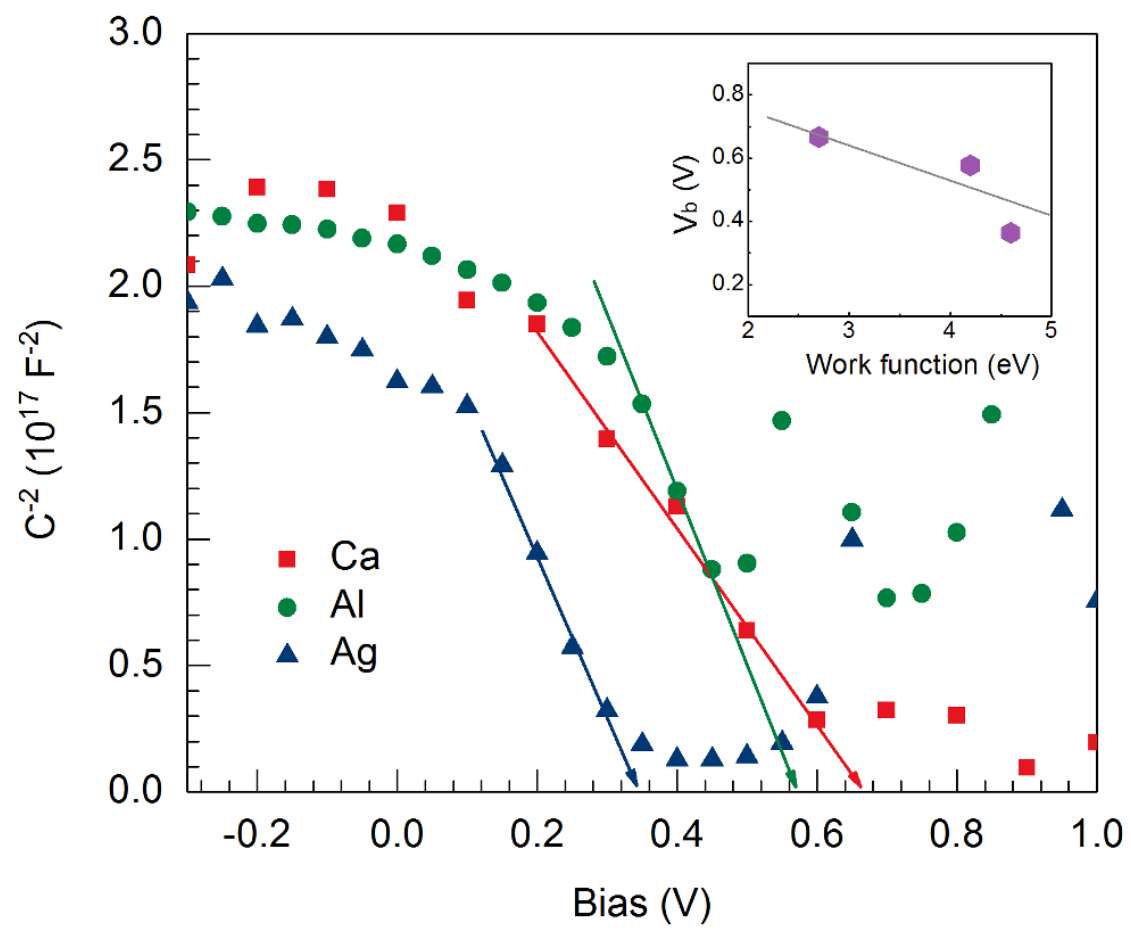

Figure 6 Built-in potential of devices with different cathode. Mott-Schottky plots of devices with different metals as cathodes. Inset shows the dependence of $\mathrm{V}_{\mathrm{b}}$ on the WF of cathodes, dashed line is the slope $\mathrm{S}$.

that of surface states. Assuming that the surface states are distributed in an interfacial layer with thickness of about $0.5 \mathrm{~nm}^{28}$, the surface states in this layer can exchange charge carriers with semiconductor and metal and withstand potential drop across itself. In the model of Bisquert, at the flat-band voltage, the depletion area in semiconductor disappears and the charges in the interfacial layer are only provided by the metal ${ }^{31}$. Therefore if we further assume that the density of the surface states is constant (Ds) between the Fermi level of metal and surface states, then Ds could be calculated with the relationship between flat-band voltages and different metal WFs, e.g., $S^{31,32}$ :

$$
\mathrm{S}=\frac{1}{1+q^{2} \delta D_{s} / \varepsilon_{i} \varepsilon_{0}}
$$

Here $\delta$ is the thickness of interfacial layer, $\varepsilon_{i}$ the dielectric constant of interfacial layer, $\varepsilon_{0}$ the permittivity of vacuum. The experimental results of Fig. 6 are summarized in the inset, the dashed line is a guide for eyes from which the slope $S$ is calculated to be $0.11 . \varepsilon_{i}$ here is 4.36 , obtained by the CV measurement under high frequency condition (details are shown in Supplementary Information). Substituting them into equation (4), Ds is deduced to be $3.9 \times 10^{14} \mathrm{~cm}^{2} \mathrm{eV}^{-1}$. From the Mott-Schottky analysis of Fig. 6, we can estimate the doping concentration $N_{A} \approx 2.7 \times 10^{16} \mathrm{~cm}^{-3}$ by:

$$
\frac{1}{C^{2}}=\frac{2\left(V_{b i}-V\right)}{A^{2} e \varepsilon \varepsilon_{0} N_{A}}
$$

where $V_{b i}$ is the built-in potential, which is equal to $V_{b}$ here ${ }^{31}, V$ is applied voltage, and $\mathrm{A}$ is the device area. Assuming the density of states at the HOMO level is about $10^{20} \mathrm{~cm}^{-3}$ and the holes occupy the states at HOMO level according to the Boltzmann distribution, the Fermi level of blend can be calculated to be about $5.0 \mathrm{eV}$ and finally the Fermi level of surface states is about $4.5 \mathrm{eV}^{31}$.
Impedance measurement and simulation. Then we apply a combined experimental and simulation approach, impedance spectroscopy (IS), to probe the dynamics of charge transfer and recombination at the interface. IS is a non-destructive tool that has been commonly used in OSCs in the past few years ${ }^{33-39}$. Because it can distinguish different processes inside the working devices according to their response to the external applied alternating current (AC) signal. The device structure used for IS measurement is ITO/PEDOT:PSS/PTB7:PC ${ }_{71} \mathrm{BM} / \mathrm{CBL}$ (w/ wo)/Al, Cole-Cole plots of the devices under 1 sun illumination and different bias are shown in Fig. 7a and b. It can be seen the impedance responses of these devices are semicircles in the complex plane with lateral axis standing for the real part of the impedance (ReZ) and vertical axis as the negative imaginary part of the impedance (-ImZ). At high frequencies (shown in the inset of Fig. 7), it is noted all the curves intersect with lateral axis at about $25 \Omega$, which represents for series resistance of the devices ${ }^{35}$. While at low frequencies, these plots intersect with lateral axis at different points which is related to recombination resistance $e^{40}$. When applied bias increases from $0.1 \mathrm{~V}$ to the vicinity of $\mathrm{V}_{\mathrm{OC}}$, recombination resistance decreases rapidly. This is because under high bias density of carriers inside the device increases significantly, as a result, more recombination events are induced.

In order to extract these constants in impedance response from different processes, equivalent circuits are often established to fit the experimental data. Fig. $8 \mathrm{a}$ and $\mathrm{b}$ show the equivalent circuits we use for devices with CBL and without CBL. $R_{0}$ is series resistance, $C_{1}$ is the geometrical capacitance of the whole device and $\mathrm{R}_{1}$ stands for the charge transport. It is well-known that paralleled combination of resistance and capacitance accounts for the recombination process happened at the interface of donor and acceptor. In the famous transmission line model, infinite $\mathrm{R} \| \mathrm{C}$ circuits are connected in series to represent for recombination processes with different relaxation time $^{36,38}$. For simplicity, we use $\mathrm{R}_{2} \| \mathrm{CPE}$ to stand for the distribution of relaxation time for different recombination processes ${ }^{35,41}$. CPE is abbreviation for constant phase element, generally used to denote non-ideal capacitor. In Fig. 8 (b) a third parallel $R_{3} \| C_{3}$ is used to stand for the recombination induced by the surface states. Fig. $8 \mathrm{c}$ 

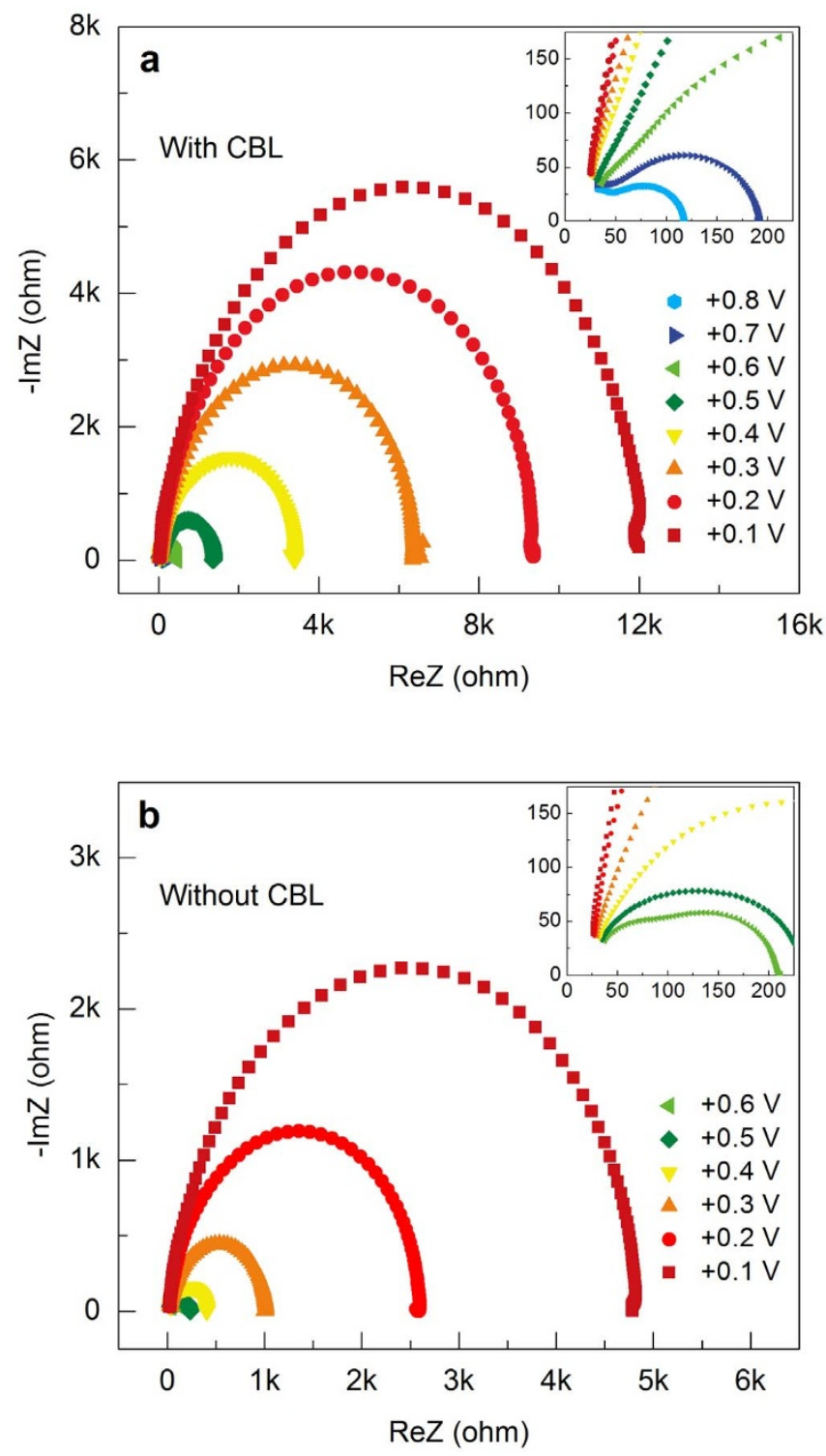

Figure 7 Impedance spectra of devices with and without CBL. Cole-Cole plots of devices under 1 sun illumination and different bias: (a) with $1.0 \mathrm{mg} / \mathrm{ml}$ NAMPC as CBL, (b) without CBL. Insets show the high frequency part of the plots from which the series resistances can be extracted.

displays the fitting results for devices with $\mathrm{CBL}$ under $+0.7 \mathrm{~V}$ bias (using model in Fig. 7a) and without CBL under $+0.5 \mathrm{~V}$ bias (using model in Fig. 7b). The biases are chosen to make the devices work around the flat-band points. It can be seen the fitting lines show good agreement with the measuring data, with the errors under $2 \%$ in all cases (provided by the built-in software of our IS equipment).

Capacitance $\mathrm{C}_{3}$ extracted from fitting is about $30 \mathrm{nF}$ from which the density of surface states can be calculated by $C_{3} /\left(q^{2} A\right)$ simply $^{28,42,43}$. Here $\mathrm{A}$ is the area of device, therefore $D_{s}=4.5 \times$ $10^{12} \mathrm{eV}^{-1} \mathrm{~cm}^{-2}$. This value is two orders of magnitude lower than the one calculated by $\mathrm{CV}$ measurement, which can be attributed to the rough assumption of parameters in the calculation, such as thickness of interfacial layer, deviation of metal WF deposited in high vacuum and in our experiments, density of surface states being constant and so on.

In addition, from impedance spectra the effective lifetime can be estimated $^{30,34}$. The peak of the large semicircle in Cole-Cole plot corresponds to a frequency, and the reciprocal of this frequency is the effective lifetime. For device with CBL under $+0.6 \mathrm{~V}$ bias and device without CBL under $+0.4 \mathrm{~V}$ (each bias is chosen in the vicinity of maximum power point), the effective lifetime is $7.7 \mu \mathrm{s}$ and $4.6 \mu \mathrm{s}$, respectively, indicating the device with $\mathrm{CBL}$ suffers less recombination.

\section{Discussion}

At last we explore the interface of CBL/cathode by UPS. The samples used are: $\mathrm{Al}, \mathrm{Al} / \mathrm{CBL}, \mathrm{Ag}, \mathrm{Ag} / \mathrm{CBL}, \mathrm{Au}$ and $\mathrm{Au} / \mathrm{CBL}$, respectively and results are displayed in Fig. $3 \mathrm{~b}$. It is noted here that the measured WFs of $\mathrm{Al}$ and $\mathrm{Au}$ are quite distinct from the common values $(4.2 \mathrm{eV}$ for $\mathrm{Al}, 5.4 \mathrm{eV}$ for $\mathrm{Au}$ ). This is because the samples are exposed to the air before they are transferred into the chamber of UPS, Al was oxidized and $2.98 \mathrm{eV}$ should be the $\mathrm{WF}$ of $\mathrm{AlO}_{\mathrm{X}}$; while the common WF of Au can be obtained when measured at clean surface in high vacuum condition, our result indicates the surface of Au layer may be soiled in the air. At this time the WFs of metals show displacement after spin-coating CBL. This indicates that dipole forms at this interface and pulls down the WF of cathode.

In Bisquert's model, $\mathrm{V}_{\mathrm{OC}}$ is determined by Fermi level difference of semiconductor and cathode (Schottky barrier). Based on our observations, it is shown however, surface states pin the Ferimi level of organic semiconductor to a certain value. This value is independent on the Fermi level of metal used as cathode. That is to say, the energy level of surface states dominates the height Schottky barrier and acts as Fermi level of cathode. Therefore even if CBL can form dipole moments at interfaces of blend/CBL and CBL/cathode, it plays a small part in the whole built-in potential.

While if surface states are suppressed effectively during the spin coating process, the Fermi level of organic semiconductor is unpinned and influence of CBL on $\mathrm{V}_{\mathrm{OC}}$ is the overall energy level shift between semiconductor and cathode induced by the dipole moments. This has also been observed by other group ${ }^{13}$.

In conclusions, we use a fullerene based polar molecule NMPAC as CBL in OSCs, and investigate its role in enhancing the device performance. It is found that the effect of CBL is originated from three aspects. Both solvent and solute of the CBL solution contribute to the optimized performance. Firstly the solvent, methanol, which is used to dissolve NMPAC, can remove the intrinsic states at the surface of the active layer. As a result, the Fermi level pinning at the surface is relieved and surface recombination is suppressed effectively. Furthermore, an additional layer of NMPAC formed at the surface of blend by spin-coating, can protect the active layer from the damage of hot atoms during cathode evaporation, and the NMPAC layer (owning deep HOMO level) can also block the holes from transferring to the cathode without hindering electrons extraction. Therefore even if deposited cathode induced states at the surface of $\mathrm{CBL}$, there would be less surface recombination due to the lack of holes. Finally the interfacial dipole moments formed at blend/CBL and/or CBL/cathode interface, alter the energy level alignment. It is very meaningful when surface states are eliminated. We believe our study here is of great importance to understanding the role of CBL in OSCs, designing new buffer layer materials and optimizing the device performance.

\section{Methods}

Device Fabrication. P3HT, PC71BM and PTB7 were purchased from Rieke Metals, Nano-C and 1-Materials, respectively. All the materials were used as received. The configuration of the devices is shown in Fig. 1. The pre-cleaned ITO glass substrate was firstly treated by oxygen plasma for $6 \mathrm{~min}$, and then a $30 \mathrm{~nm}$ PEDOT:PSS (poly (3, 4-ethylenedioxythio-phene):poly(styrenesulfonate), Clevios P VPAI 4083, from H. C. Starck) layer was spin-coated on the substrate, followed by thermal treatment at $140^{\circ} \mathrm{C}$ for $10 \mathrm{~min}$ on a hotplate. The substrate was then transferred into the nitrogen glove box, and all the other processes were done in the glove box unless otherwise stated. PTB7:PC71BM were dissolved in o-dichlorobenzene (o-DCB) to be the active layer with the ratio of $10: 15 \mathrm{mg} / \mathrm{mL}$, with $3 \%$ volume 1,8 -diiodooctane as the additive. Then the blend was spin-coated onto the substrate at $900 \mathrm{rpm}$ for $90 \mathrm{~s}$. NMPAC was dissolved in methanol with varied concentrations and spin-coated atop 

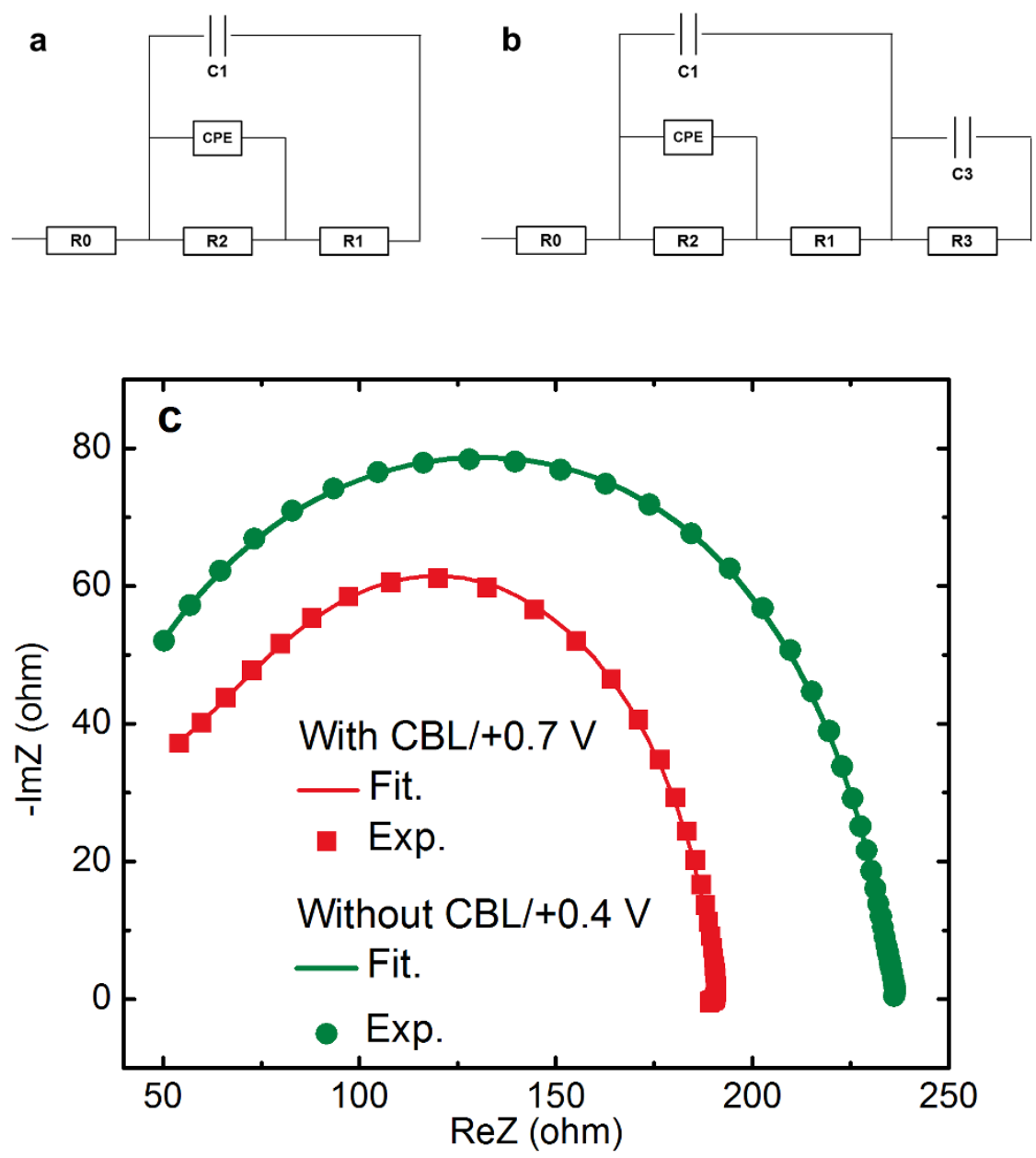

Figure 8 Simulations by fitting impedance data into device model. Equivalent circuits for device (a) with CBL and (b) without CBL, and the fitting results obtained by these two models.

the active layer at 3,000 rpm for $30 \mathrm{~s}$. Finally, top electrodes were deposited in vacuum at a pressure of about $5.0 \times 10^{-5} \mathrm{~Pa}$. The active device area was $4.2 \mathrm{~mm}^{2}$.

The thickness of the interlayer was determined using a Profilometer, combined with extrapolation from an absorbance-thickness curve which assumed a linear dependence of absorbance at $320 \mathrm{~nm}$ for NMPAC.

J-V Characterization and EQE. Current density-voltage (J-V) characteristics of the devices were measured with a computer-controlled Keithley 2400 source meter and Newport $6279 \mathrm{NS}$ solar simulator $(450 \mathrm{~W})$ with $100 \mathrm{~mW} / \mathrm{cm}^{2}$ illumination. The external quantum efficiency (EQE) spectra were collected using Oriel IQE-200 ${ }^{\mathrm{TM}}$ in the atmosphere. UPS spectra were obtained using a He discharged lamp (He I $21.22 \mathrm{eV})$.

$\mathrm{CV}$ and IS measurements. The capacitance-voltage (CV) and impedance spectroscopy (IS) measurements were performed using a Zahner Zennium electrochemical workstation. The CV measurements were recorded at a frequency of $1 \mathrm{kHz}$ for extracting $\mathrm{V}_{\mathrm{b}}$ and $\mathrm{N}_{\mathrm{A}}$, and at a frequency of $1 \mathrm{MHz}$ to calculate the dielectric constant of blend. The impedance spectra were recorded by applying varied AC signal from $10 \mathrm{~Hz}$ to $1 \mathrm{MHz}$. All the AC oscillating amplitudes were set as low as $10 \mathrm{mV}$ (rms) to maintain the linearity of the response.

1. Huang, F., Wu, H. \& Cao, Y. Water/alcohol soluble conjugated polymers as highly efficient electron transporting/injection layer in optoelectronic devices. Chem. Soc. Rev. 39, 2500-2521 (2010).

2. Po, R., Carbonera, C., Bernardi, A. \& Camaioni, N. The role of buffer layers in polymer solar cells. Energy Environ. Sci. 4, 285 (2011)

3. Yip, H.-L. \& Jen, A. K. Y. Recent advances in solution-processed interfacial materials for efficient and stable polymer solar cells. Energy Environ. Sci. 5, 5994-6011 (2012)

4. Zhicai, H. et al. Enhanced power-conversion efficiency in polymer solar cells using an inverted device structure. Nat. Photonics 6, 591-595 (2012).

5. Yang, T. et al. Inverted polymer solar cells with $8.4 \%$ efficiency by conjugated polyelectrolyte. Energy Environ. Sci. 5, 8208-8214 (2012).
6. Chang, Y.-M. et al. Electrostatic Self-Assembly Conjugated PolyelectrolyteSurfactant Complex as an Interlayer for High Performance Polymer Solar Cells. Adv. Funct. Mater. 22, 3284-3289 (2012).

7. He, Z. et al. Simultaneous Enhancement of Open-Circuit Voltage, Short-Circuit Current Density, and Fill Factor in Polymer Solar Cells. Adv. Mater. 23, 4636-4643 (2011).

8. Zhang, Z.-G. et al. Poly(ethylene glycol) modified [60] fullerene as electron buffer layer for high-performance polymer solar cells. Appl. Phys. Lett. 102, 143902 (2013).

9. Zhou, Y. et al. A Universal Method to Produce Low-Work Function Electrodes for Organic Electronics. Science 336, 327-332 (2012).

10. Nam, S. et al. Effects of direct solvent exposure on the nanoscale morphologies and electrical characteristics of PCBM-based transistors and photovoltaics. J. Mater. Chem. 22, 5543-5549 (2012).

11. Liu, X., Wen, W. \& Bazan, G. C. Post-Deposition Treatment of an ArylatedCarbazole Conjugated Polymer for Solar Cell Fabrication. Adv. Mater. 24, 4505-4510 (2012).

12. Li, H. et al. Solvent-soaking treatment induced morphology evolution in P3HT/ PCBM composite films. J. Mater. Chem. 21, 6563-6568 (2011).

13. Lv, M. et al. A Hyperbranched Conjugated Polymer as the Cathode Interlayer for High-Performance Polymer Solar Cells. Adv. Mater. 25, 6889-6894 (2013).

14. Zhou, H. et al. High-Efficiency Polymer Solar Cells Enhanced by Solvent Treatment. Adv. Mater. 25, 1646-1652 (2013).

15. Isobe, H., Tomita, N. \& Nakamura, E. One-Step Multiple Addition of Amine to [60] Fullerene. Synthesis of Tetra(amino)fullerene Epoxide under Photochemical Aerobic Conditions. Org. Lett. 2, 3663-3665 (2000).

16. Isobe, H., Tanaka, T., Nakanishi, W., Lemiègre, L. \& Nakamura, E. Regioselective Oxygenative Tetraamination of [60] Fullerene. Fullerene-mediated Reduction of Molecular Oxygen by Amine via Ground State Single Electron Transfer in Dimethyl Sulfoxide. J. Org. Chem. 70, 4826-4832 (2005).

17. Jung, J. W., Jo, J. W. \& Jo, W. H. Enhanced performance and air stability of polymer solar cells by formation of a self-assembled buffer layer from fullereneend-capped poly(ethylene glycol). Adv. Mater. 23, 1782-1787 (2011).

18. O'Malley, K. M., Li, C.-Z., Yip, H.-L. \& Jen, A. K. Y. Enhanced Open-Circuit Voltage in High Performance Polymer/Fullerene Bulk-Heterojunction Solar Cells 
by Cathode Modification with a C60 Surfactant. Adv. Energy Mater. 2, 82-86 (2012).

19. Li, C.-Z. et al. Effective interfacial layer to enhance efficiency of polymer solar cells via solution-processed fullerene-surfactants. J. Mater. Chem. 22, 8574 (2012).

20. Vandewal, K., Tvingstedt, K., Gadisa, A., Inganas, O. \& Manca, J. V. On the origin of the open-circuit voltage of polymer-fullerene solar cells. Nat. Mater. 8, 904-909 (2009).

21. Potscavage, W. J., Yoo, S. \& Kippelen, B. Origin of the open-circuit voltage in multilayer heterojunction organic solar cells. Appl. Phys. Lett. 93, 193308 (2008).

22. Qi, B. \& Wang, J. Open-circuit voltage in organic solar cells. J. Mater. Chem. 22, 24315-24325 (2012).

23. Şahin, Y., Alem, S., de Bettignies, R. \& Nunzi, J.-M. Development of air stable polymer solar cells using an inverted gold on top anode structure. Thin Solid Films 476, 340-343 (2005).

24. Shrotriya, V., Li, G., Yao, Y., Chu, C.-W. \& Yang, Y. Transition metal oxides as the buffer layer for polymer photovoltaic cells. Appl. Phys. Lett. 88, 073508 (2006).

25. Hayakawa, A., Yoshikawa, O., Fujieda, T., Uehara, K. \& Yoshikawa, S. High performance polythiophene/fullerene bulk-heterojunction solar cell with a TiOx hole blocking layer. Appl. Phys. Lett. 90, 163517 (2007).

26. Qi, B. \& Wang, J. Fill factor in organic solar cells. Phys. Chem. Chem. Phys. 15 , 8972-8982 (2013)

27. Sze, S. M. \& Ng, K. K. Physics of Semiconductor Devices (John Wiley \& Sons, Inc., 2006).

28. Kelly, J. J. \& Memming, R. The Influence of Surface Recombination and Trapping on the Cathodic Photocurrent at p-Type III-V Electrodes. J. Electrochem. Soc. 129, 730-738 (1982).

29. Bisquert, J. et al. Band unpinning and photovoltaic model for P3HT:PCBM organic bulk heterojunctions under illumination. Chem. Phys. Lett. 465, 57-62 (2008).

30. Garcia-Belmonte, G. et al. Charge carrier mobility and lifetime of organic bulk heterojunctions analyzed by impedance spectroscopy. Org. Electron. 9, 847-851 (2008)

31. Guerrero, A. et al. How the Charge-Neutrality Level of Interface States Controls Energy Level Alignment in Cathode Contacts of Organic Bulk-Heterojunction Solar Cells. ACS Nano 6, 3453-3460 (2012).

32. Cowley, A. M. Depletion Capacitance and Diffusion Potential of Gallium Phosphide Schottky-Barrier Diodes. J. Appl. Phys. 37, 3024-3032 (1966)

33. Fabregat-Santiago, F., Garcia-Belmonte, G., Mora-Sero, I. \& Bisquert, J. Characterization of nanostructured hybrid and organic solar cells by impedance spectroscopy. Phys. Chem. Chem. Phys. 13, 9083-9118 (2011).

34. Garcia-Belmonte, G., Boix, P. P., Bisquert, J., Sessolo, M. \& Bolink, H. J. Simultaneous determination of carrier lifetime and electron density-of-states in P3HT:PCBM organic solar cells under illumination by impedance spectroscopy. Sol. Energy Mater. Sol. Cells 94, 366-375 (2010).

35. Leever, B. J., Bailey, C. A., Marks, T. J., Hersam, M. C. \& Durstock, M. F. In Situ Characterization of Lifetime and Morphology in Operating Bulk Heterojunction Organic Photovoltaic Devices by Impedance Spectroscopy. Adv. Energy Mater. 2, 120-128 (2012).

36. Bisquert, J. Beyond the quasistatic approximation: Impedance and capacitance of an exponential distribution of traps. Phys. Rev. B 77, 235203 (2008).
37. Bisquert, J. \& Garcia-Belmonte, G. On Voltage, Photovoltage, and Photocurrent in Bulk Heterojunction Organic Solar Cells. J. Phys. Chem. Lett. 2, 1950-1964 (2011).

38. Garcia-Belmonte, G., Guerrero, A. \& Bisquert, J. Elucidating Operating Modes of Bulk-Heterojunction Solar Cells from Impedance Spectroscopy Analysis. J. Phys. Chem. Lett. 4, 877-886 (2013).

39. Leong, W. L., Cowan, S. R. \& Heeger, A. J. Differential Resistance Analysis of Charge Carrier Losses in Organic Bulk Heterojunction Solar Cells: Observing the Transition from Bimolecular to Trap-Assisted Recombination and Quantifying the Order of Recombination. Adv. Energy Mater. 1, 517-522 (2011).

40. Bisquert, J. Theory of the Impedance of Electron Diffusion and Recombination in a Thin Layer. J. Phys. Chem. B 106, 325-333 (2001).

41. Raistrick, I. D., Franceschetti, D. R. \& Macdonald, J. R. in Impedance Spectroscopy 27-128 (John Wiley \& Sons, Inc. 2005).

42. Gerischer, H. Photoassisted interfacial electron transfer. Surf. Sci. 101, 518-530 (1980).

43. Ripolles-Sanchis, T., Guerrero, A., Bisquert, J. \& Garcia-Belmonte, G. Diffusion Recombination Determines Collected Current and Voltage in Polymer:Fullerene Solar Cells. J. Phys. Chem. C 116, 16925-16933 (2012).

\section{Acknowledgments}

The authors acknowledge the financial support by 973 Program (Grant No. 2014CB643600, 2014CB643503 and 2011CB932304), National Natural Science Foundation of China (61405208), the CAS/SAFEA International Partnership Program for Creative Research Teams and the Strategic Priority Research Program of the Chinese Academy of Sciences Grant No. XDB12030200).

\section{Author contributions}

Z.Z. synthesized the NMPAC and performed cyclic voltammograms experiments, B.Q. performed the experiments and data analysis, B.Q., Z.Z. and J.W. wrote the paper, J.W. supervised the project.

\section{Additional information}

Supplementary information accompanies this paper at http://www.nature.com/ scientificreports

Competing financial interests: The authors declare no competing financial interests.

How to cite this article: Qi, B., Zhang, Z.-G. \& Wang, J. Uncovering the role of cathode buffer layer in organic solar cells. Sci. Rep. 5, 7803; DOI:10.1038/srep07803 (2015).

This work is licensed under a Creative Commons Attribution-NonCommercialNoDerivs 4.0 International License. The images or other third party material in this article are included in the article's Creative Commons license, unless indicated otherwise in the credit line; if the material is not included under the Creative Commons license, users will need to obtain permission from the license holder in order to reproduce the material. To view a copy of this license, visit http:// creativecommons.org/licenses/by-nc-nd/4.0/ 